\title{
Exosomes as nanocarriers for siRNA delivery: paradigms and challenges
}

\author{
Fahimeh Shahabipour ${ }^{1,2}$, Maciej Banach ${ }^{3}$, Amirhossein Sahebkar ${ }^{4}$
}

\author{
${ }^{1}$ National Cell Bank of Iran, Pasteur Institute of Iran, Tehran, Iran \\ ${ }^{2}$ Neurogenic Inflammation Research Center, Department of Medical Biotechnology, \\ Mashhad University of Medical Sciences, Mashhad, Iran \\ ${ }^{3}$ Department of Hypertension, Chair of Nephrology and Hypertension, \\ Medical University of Lodz, Lodz, Poland \\ ${ }^{4}$ Biotechnology Research Center, Mashhad University of Medical Sciences, Mashhad, \\ Iran
}

Submitted: 13 March 2016

Accepted: 18 March 2016

Arch Med Sci 2016; 12, 6: 1324-1326

DOI: $10.5114 /$ aoms.2016.62911

Copyright $\odot 2016$ Termedia \& Banach

\begin{abstract}
Exosomes are nano-sized vesicles that facilitate intercellular communications through carrying genetic materials and functional biomolecules. Owing to their unique size and structure, exosomes have emerged as a useful tool to overcome the limitations of siRNA delivery. The use of exosomes as siRNA delivery vehicles lacks certain disadvantages of the existing foreign delivery systems such as viruses, polycationic polymers and liposomes, and introduces several advantages including inherent capacity to pass through biological barriers and escape from phagocytosis by the reticuloendothelial system, as well as being biocompatible, non-toxic, and immunologically inert. Different strategies have been employed to harness exosome-based delivery systems, including surface modification with targeting ligands, and using exosome-display technology, virus-modified exosomes, and exosome-mimetic vesicles. The present review provides a capsule summary of the recent advances and current challenges in the field of exosome-mediated siRNA delivery.
\end{abstract}

Key words: exosome, siRNA, microRNA, nanomedicine, drug delivery, gene therapy.

Exosomes are naturally occurring nanosized extracellular vesicles ranging from 30 to $120 \mathrm{~nm}$ in diameter that originate from many cell types such as dendritic cells, B cells, T cells, mast cells, epithelial cells and tumor cells [1]. Fusion of multivesicular bodies - that are formed by inward budding of late endosomes - with plasma membrane releases exosomes to the extracellular milieu [2]. Exosomes are composed of lipids, proteins and nucleic acids, mainly miRNAs and mRNA, though the presence of genomic and mitochondrial DNA has also been reported [3]. The main function of these vesicles is to facilitate intercellular communications through carrying shuttle RNAs, receptors and enzymes between the neighboring cells $[4,5]$. These communications are crucial for several biologically important processes, e.g. antigen presentation to T cells, signal transduction and development of immune tolerance $[1,6]$.

Owing to their unique size, structural and functional properties, exosomes have emerged as a novel delivery system for biological therapeutics and diagnostic molecules including siRNAs and recombinant

\author{
Corresponding author: \\ Amirhossein Sahebkar PhD \\ Biotechnology \\ Research Center \\ Mashhad University \\ of Medical Sciences \\ Vakilabad blvd. \\ 9177948564 Mashhad, Iran \\ Phone: +989151221496 \\ Fax: +985138002287 \\ E-mail: sahebkara@ \\ mums.ac.ir
}


proteins (Figure 1), especially those targeted to the brain tissue that require passage through the blood brain barrier. Normally, delivery of siRNAs is limited by rapid degradation, insufficient cellular uptake, inability to pass through biological barriers, and targeting to undesired cells and tissues resulting in off-target gene silencing and toxicity $[2,7]$. Furthermore, some sequence motifs of siRNAs may elicit an immune response [8]. The use of exosomes as siRNA delivery vehicles lacks certain disadvantages of the existing foreign delivery systems such as viruses, polycationic polymers (e.g. polyethylenimine (PEI)-based particles) and liposomes. Although virus-mediated siRNA delivery leads to long-term gene silencing in the target tissue, viral vectors have major problems including toxicity and immunostimulation. With respect to synthetic carrier systems such as PEI nanoparticles, delivery is mostly confined to specific tissues due to accumulation of PEI-RNA complexes in the liver, spleen and kidneys [9, 10]. In contrast, exosomes are endowed with several advantages including the inherent capacity to pass through biological barriers and escape from phagocytosis by the reticuloendothelial system, and being biocompatible, non-toxic, and immunologically inert, particularly when derived from autologous dendritic cells and monocytes [2, 10, 11]. Moreover, the capacity of exosomes to fuse with plasma membrane (mediated through surface CD55 and CD59 receptors) enables direct delivery of intravesicular cargo to the cytosol, thereby obviating the need for endosomal escape manipulations. Such surface receptors also contribute to reduced interaction of exosomes with opsonins, coagulation factors and complement proteins in the circulation and enhance the stability of the particles $[10,11]$. Owing to these unique characteristics, different strategies have been employed to develop exosome-based delivery systems. The first approach was based on the expression of exosomal membrane proteins fused to tissue-targeting molecules on the surface of secreting exosomes to trigger siRNAs specifically to the desired tissue. Using this strategy, targeted delivery of siRNAs to brain tissue has been successfully achieved with an exosomal vehicle on which a rabies virus glycoprotein (RVG)-derived peptide was fused to an integral exosomal membrane protein, Lamp2b [12]. Exosome-display technology is another viable strategy that involves specific fusion of antigens to the C1C2 domain of the lactadherin protein, which binds to phosphatidylserine and is exposed on the surface of the exosome. These constructs enhance the presentation of antigens to the immune system and are thus appropriate for vaccination purposes [7, 13]. This technique could also be used to deliver siRNAs to human mononuclear

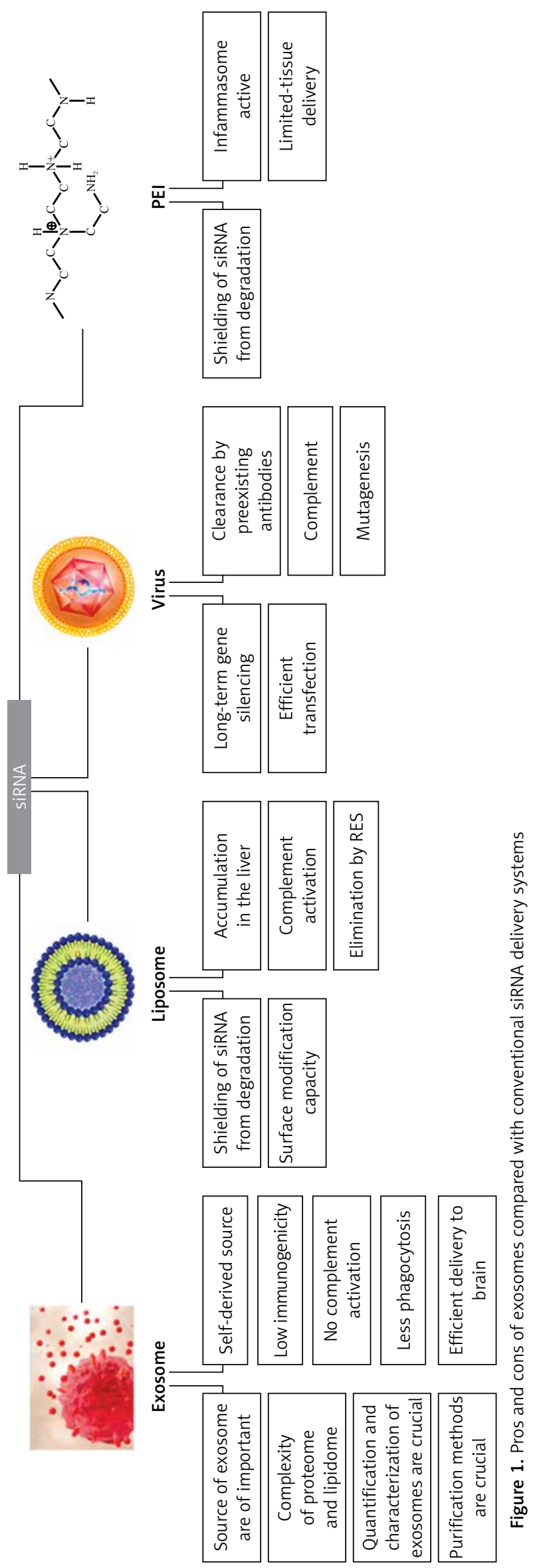


blood cells $[6,14]$. A recent alternative strategy has been described to improve exosome-mediated siRNA delivery via displaying cell-penetrating peptides (CPPs) associated with the Lamp2b protein on the surface of exosomes to enhance cellular translocation and shield the negative charge of siRNAs [15]. Development of virus-modified exosomes is another technique to increase the targeting efficiency of siRNAs in vivo. Owing to the similarities in composition, function and release characteristics between exosomes and viruses, incorporation of viral components into the endogenously produced exosome structures has been suggested as an approach to enhance siRNA delivery. Successful delivery of genetic materials by virus-modified exosomes originating from certain virus infected cells has been reported [16]. Tailoring siRNA loading in exosomes could be achieved through transfection of source cells with a plasmid encoding exosomal proteins such as Lamp2b followed by electroporation of purified modified exosomes to load siRNA [12]. However, electroporation has a low loading efficiency and may cause aggregation of siRNA molecules. Post-release chemical treatment of exosomes with Lipofectamine has the same limitations as electroporation, and efficient exosome loading techniques are yet to be introduced [17] In spite of promises, a number of challenges to the successful implementation of exosome-based siRNA delivery systems need to be addressed. The most important ones include optimization of purification and characterization methods to increase the homogeneity of exosomes and eliminate non-exosomal cellular vesicles and complexes, and tailoring transfection strategies to allow efficient loading of siRNA in exosomes [18]. Development of synthetic cell-derived exosome-mimetic nanovesicles has been proposed as a solution to overcome the problem of particle heterogeneity and low yield of exosome production [17]. Bio-inspired exosome mimetics could be manufactured by serial extrusion of monocytes and macrophage through filters with diminishing pore sizes of 10, 5 and $1 \mu \mathrm{m}$ [4]. Liposomes harboring functional components of exosomes have been the most widely studied structures to serve as exosome mimetics, with improved homogeneity and characterization capacity compared with their natural counterparts. However, much more data from proteomic and lipidomic studies are still required to identify the components that are crucial for biological functioning of exosomes [7]. Finally, the efficiency of exosome-mimetic nanovesicles versus autologous and non-autologous exosomes requires further in vivo investigations [18].

\section{Conflict of interest}

The authors declare no conflict of interest.

\section{References}

1. Vlassov AV, Magdaleno S, Setterquist R, Conrad R. Exosomes: current knowledge of their composition, biological functions, and diagnostic and therapeutic potentials. Biochim Biophys Acta 2012; 1820: 940-8.

2. Shtam TA, Kovalev RA, Varfolomeeva EY, Makarov EM, Kil YV, Filatov MV. Exosomes are natural carriers of exogenous siRNA to human cells in vitro. Cell Commun Signal 2013; 11: 88

3. Urbanelli L, Buratta S, Sagini K, Ferrara G, Lanni M, Emiliani $C$. Exosome based strategies for diagnosis and therapy. Recent Pat CNS Drug Discov 2015; 10: 10-27.

4. Jang SC, Kim OY, Yoon CM, et al. Bioinspired exosome-mimetic nanovesicles for targeted delivery of chemotherapeutics to malignant tumors. ACS Nano 2013; 7: 7698-710.

5. Valadi H, Ekström K, Bossios A, Sjöstrand M, Lee JJ, Lötvall JO. Exosome-mediated transfer of mRNAs and microRNAs is a novel mechanism of genetic exchange between cells. Nature Cell Biol 2007; 9: 654-9.

6. Théry C, Duban L, Segura E, Véron P, Lantz O, Amigorena $S$. Indirect activation of naïve CD4+ T cells by dendritic cell-derived exosomes. Nature Immunol 2002; 3 : 1156-62.

7. Kooijmans SA, Vader P, van Dommelen SM, van Solinge WW, Schiffelers RM. Exosome mimetics: a novel class of drug delivery systems. Int J Nanomed 2012; 7: 1525-41.

8. Castanotto D, Rossi JJ. The promises and pitfalls of RNA-interference-based therapeutics. Nature 2009; 457: 426-33.

9. Escors D, Breckpot K. Lentiviral vectors in gene therapy: their current status and future potential. Arch Ther Exp (Warsz) 2010; 58: 107-19.

10. van den Boorn JG, Schlee M, Coch C, Hartmann G. SiRNA delivery with exosome nanoparticles. Nature Biotechnol 2011; 29: 325-6.

11. Clayton A, Harris CL, Court J, Mason MD, Morgan BP. Antigen-presenting cell exosomes are protected from complement-mediated lysis by expression of CD55 and CD59. Eur J Immunol 2003; 33: 522-31.

12. Alvarez-Erviti L, Seow Y, Yin H, Betts C, Lakhal S, Wood MJ. Delivery of siRNA to the mouse brain by systemic injection of targeted exosomes. Nature Biotechnol 2011; 29: 341-5.

13. Hartman ZC, Wei J, Glass OK, et al. Increasing vaccine potency through exosome antigen targeting. Vaccine 2011; 29: 9361-7.

14. Wahlgren J, De L Karlson T, Brisslert $M$, et al. Plasma exosomes can deliver exogenous short interfering RNA to monocytes and lymphocytes. Nucleic Acids Res 2012; 40: e130.

15. El-Andaloussi S, Lee Y, Lakhal-Littleton S, et al. Exosomemediated delivery of siRNA in vitro and in vivo. Nat Protoc 2012; 7: 2112-26.

16. Koppers-Lalic D, Hogenboom MM, Middeldorp JM, Pegtel DM. Virus-modified exosomes for targeted RNA delivery: a new approach in nanomedicine. Adv Drug Deliv Rev 2013; 65: 348-56.

17. Lässer C. Exosomes in diagnostic and therapeutic applications: biomarker, vaccine and RNA interference delivery vehicle. Expert Opin Biol Ther 2015; 15: 103-17.

18. Jang SC, Gho YS. Could bioengineered exosome-mimetic nanovesicles be an efficient strategy for the delivery of chemotherapeutics? Nanomedicine 2014; 9: 177-80. 